\title{
SYMPLECTIC TOMOGRAPHY OF NONCLASSICAL STATES OF TRAPPED ION
}

\author{
Olga Man'ko円 \\ International Center for Theoretical Physics, Trieste, Italy
}

\begin{abstract}
The marginal distribution of squeezed and rotated quadrature for two types of nonclassical states of trapped ion - for squeezed and correlated states and for squeezed even and odd coherent states (squeezed Schrödinger cat states) is studied. The obtained marginal distribution for the two types of states is shown to satisfy classical dynamical equation equivalent to standard quantum evolution equation for density matrix (wave function) derived in symplectic tomography scheme.
\end{abstract}

\section{Introduction}

Recently, in [1] it was shown that the steady state of trapped ion irradiated by bichromatic laser field is superposition of two coherent states [2] which is even/odd coherent state introduced in [3] and interpreted in [4] for large amplitudes of the superposition partners as Schrödinger cat states [5]. The theory of ion in the Paul trap was developed in [6, 7], where the trapped ion was described by the model of quantum oscillator with periodically varying frequency. Gaussian packets, discrete modes and propagator of such oscillator have been obtained in 8 . Linear in position and momentum integrals of motion have been found for the oscillator in [6, 7, 9]. In [10], on the basis of analysis [11], the procedure was formulated to obtain the Wigner function of quantum system in terms of marginal distribution of rotated quadrature which may be measured by balanced homodyne detector. The scheme which was called optical tomography has been used experimentally [12]. In [13], the symplectic tomography procedure has been suggested in which measuring the quantum states was proposed by means of measuring marginal distribution for squeezed, rotated and shifted quadrature.

In [14], a new equation in quantum mechanics was introduced describing time evolution of this marginal distribution. The equation has completely classical form but contains all information about quantum system.

The aim of this work is to consider two important types of nonclassical states of the trapped ion. First we consider squeezed and correlated states [16] of the ion in the Paul trap [6, 7]. Then we study also even and odd coherent states of the ion irradiated by bichromatic laser field [1]. The states of trapped ion are investigated in frame of symplectic tomography procedure suggested in [13] and using the new quantum evolution equation of [14]. It is worthy to note that in 15] endoscopy procedure to measure the quantum states of the trapped ion is elaborated in detail.

\footnotetext{
${ }^{1}$ On leave from Lebedev Physical Institute, Moscow, Russia. Contribution to the Adriatico Research Conference "Interferometry 2" (ICTP, Trieste, 1-11 March, 1996), originally submitted in English 13 March, 1996.
} 


\section{Gaussian Wigner Function}

The generic mixed squeezed state of the trapped ion with the density operator $\hat{\rho}$ is described by the Wigner function $W(p, q)$ of the Gaussian form which contains five real parameters [17] (the one-mode oscillator case). Two parameters are mean values of momentum $\langle p\rangle$ and position $\langle q\rangle$ and other three parameters are matrix elements of the real dispersion matrix $m$ :

$$
\begin{aligned}
& m_{11}=\sigma_{p p} ; \\
& m_{12}=\sigma_{p q} ; \\
& m_{22}=\sigma_{q q} .
\end{aligned}
$$

Below, we will use the invariant parameters

$$
T=\operatorname{Tr} m=\sigma_{p p}+\sigma_{q q}
$$

and

$$
d=\operatorname{det} m=\sigma_{p p} \sigma_{q q}-\sigma_{p q}^{2} .
$$

For one-mode system, the generic Gaussian Wigner function has the form (see, for example, [17, 18 )

$$
W(p, q)=\frac{1}{\sqrt{d}} \exp \left\{-\frac{1}{2 d}\left[\sigma_{q q}(p-\langle p\rangle)^{2}+\sigma_{p p}(q-\langle q\rangle)^{2}-2 \sigma_{p q}(p-\langle p\rangle)(q-\langle q\rangle)\right]\right\} .
$$

The parameters $\langle p\rangle$ and $\langle q\rangle$ are given by the formulas

$$
\begin{aligned}
& \langle p\rangle=\operatorname{Tr} \hat{\rho} \hat{p} \\
& \langle q\rangle=\operatorname{Tr} \hat{\rho} \hat{q},
\end{aligned}
$$

where the operators $\hat{p}$ and $\hat{q}$ are the quadrature components of creation $a^{\dagger}$ and annihilation $a$ operators

$$
\begin{gathered}
\hat{p}=\frac{a-a^{\dagger}}{i \sqrt{2}} \\
\hat{q}=\frac{a+a^{\dagger}}{\sqrt{2}} .
\end{gathered}
$$

The matrix elements of the real symmetric dispersion matrix $m$ are defined as follows

$$
\begin{aligned}
\sigma_{p p} & =\operatorname{Tr} \hat{\rho} \hat{p}^{2}-\langle p\rangle^{2} ; \\
\sigma_{q q} & =\operatorname{Tr} \hat{\rho} \hat{q}^{2}-\langle q\rangle^{2} ; \\
\sigma_{p q} & =\frac{1}{2} \operatorname{Tr} \hat{\rho}(\hat{p} \hat{q}+\hat{q} \hat{p})-\langle p\rangle\langle q\rangle .
\end{aligned}
$$

Due to the physical meaning of the dispersions, the parameters $\sigma_{p p}$ and $\sigma_{q q}$ must be nonnegative numbers, so the invariant parameter $T$ (2) is a positive number. Also the determinant $d$ (3) of the dispersion matrix must be positive. For a pure Gaussian state, the parameter $d=1 / 4$. 


\section{Squeezed States of Trapped Ion}

Since the ion in the Paul trap is described by the model of parametric oscillator in this section we review its properties. For the parametric oscillator with arbitrary time dependence of the frequency and the Hamiltonian

$$
H=-\frac{\partial^{2}}{2 \partial x^{2}}+\frac{\omega^{2}(t) x^{2}}{2},
$$

where we put $\hbar=m=\omega(0)=1$ and used expressions for the position and momentum operators in the coordinate representation, there exists the time-dependent integral of motion found in [9]

$$
A=\frac{i}{\sqrt{2}}[\varepsilon(t) \hat{p}-\dot{\varepsilon}(t) \hat{q}],
$$

where

$$
\begin{aligned}
\ddot{\varepsilon}(t)+\omega^{2}(t) \varepsilon(t) & =0 ; \\
\varepsilon(0) & =1 ; \\
\dot{\varepsilon}(0) & =i,
\end{aligned}
$$

satisfying the commutation relation

$$
\left[A, A^{\dagger}\right]=1 \text {. }
$$

For the trapped ion, the time-dependence of frequency is taken to be periodical one [6]

$$
\omega^{2}(t)=1+\kappa^{2} \sin ^{2} \Omega t .
$$

It is easy to show that packet solutions to the Schrödinger equation may be introduced and interpreted as coherent states [9], since they are eigenstates of the operator $A$ (11), of the form

$$
\Psi_{\alpha}(x, t)=\Psi_{0}(x, t) \exp \left\{-\frac{|\alpha|^{2}}{2}-\frac{\alpha^{2} \varepsilon^{*}(t)}{2 \varepsilon(t)}+\frac{\sqrt{2} \alpha x}{\varepsilon}\right\},
$$

where

$$
\Psi_{0}(x, t)=\pi^{-1 / 4}[\varepsilon(t)]^{-1 / 2} \exp \frac{i \dot{\varepsilon}(t) x^{2}}{2 \varepsilon(t)}
$$

is analog of the ground state of the oscillator and $\alpha$ is a complex number. Variances of the position and momentum of the parametric oscillator in the state (16) are

$$
\begin{aligned}
\sigma_{q q} & =\frac{|\varepsilon(t)|^{2}}{2} ; \\
\sigma_{p p} & =\frac{|\dot{\varepsilon}(t)|^{2}}{2},
\end{aligned}
$$

and the correlation coefficient $r$ of the position and momentum has the value corresponding to minimization of the Schrödinger uncertainty relation [19]

$$
\begin{aligned}
\sigma_{q q} \sigma_{p p} & =\frac{1}{4} \frac{1}{1-r^{2}} \\
r & =\frac{\sigma_{p q}}{\sqrt{\sigma_{q q} \sigma_{p p}}} .
\end{aligned}
$$


If $\sigma_{q q}<1 / 2\left(\sigma_{p p}<1 / 2\right)$ we have squeezing in quadrature components.

The analogs of orthogonal and complete system of number states which are excited states of the ion in the Paul trap are obtained by expansion of (15) into power series in $\alpha$. We have

$$
\Psi_{m}(x, t)=\left(\frac{\varepsilon^{*}(t)}{2 \varepsilon(t)}\right)^{m / 2} \frac{1}{\sqrt{m !}} \Psi_{0}(x, t) H_{m}\left(\frac{x}{|\varepsilon(t)|}\right)
$$

and these squeezed and correlated number states are eigenstates of the invariant $A^{\dagger} A$.

Another normalized solution to the Schrödinger equation

$$
\Psi_{\alpha}^{(+)}(x, t)=2 N^{(+)} \Psi_{0}(x, t) \exp \left\{-\frac{|\alpha|^{2}}{2}-\frac{\varepsilon^{*}(t) \alpha^{2}}{2 \varepsilon(t)}\right\} \cosh \frac{\sqrt{2} \alpha x}{\varepsilon(t)}
$$

where

$$
N^{(+)}=\frac{\exp \left(|\alpha|^{2} / 2\right)}{2 \sqrt{\cosh |\alpha|^{2}}}
$$

is the squeezed even coherent state [3] (the squeezed Schrödinger cat state). The odd coherent state of the parametric oscillator

$$
\Psi_{\alpha}^{(-)}(x, t)=2 N^{(-)} \Psi_{0}(x, t) \exp \left\{-\frac{|\alpha|^{2}}{2}-\frac{\varepsilon^{*}(t) \alpha^{2}}{2 \varepsilon(t)}\right\} \sinh \frac{\sqrt{2} \alpha x}{\varepsilon(t)}
$$

where

$$
N^{(-)}=\frac{\exp \left(|\alpha|^{2} / 2\right)}{2 \sqrt{\sinh |\alpha|^{2}}}
$$

satisfies the Schrödinger equation and is the eigenstate of the integral of motion $A^{2}$ (as well as the even coherent state) with the eigenvalue $\alpha^{2}$. These states are one-mode examples of squeezed and correlated Schrödinger cat states constructed in [20].

\section{Tomography of Trapped Ion}

In [13], it was shown that for the generic linear combination of quadratures which is a measurable observable $(\hbar=1)$

$$
\widehat{X}=\mu \hat{q}+\nu \hat{p}+\delta
$$

where $\hat{q}$ and $\hat{p}$ are the position and momentum, respectively, the marginal distribution $w(X, \mu, \nu, \delta)$ (normalized with respect to the $X$ variable), depending upon three extra real parameters $\mu ; \nu ; \delta$, is related to the state of the quantum system expressed in terms of its Wigner function $W(q, p)$ as follows

$$
w(X, \mu, \nu, \delta)=\int \exp [-i k(X-\mu q-\nu p-\delta)] W(q, p) \frac{d k d q d p}{(2 \pi)^{2}}
$$

As it follows from this formula, the marginal distribution depends on the difference of the variables

$$
X-\delta=Y .
$$


So, we could introduce

$$
P(Y, \mu, \nu)=w(X, \mu, \nu, \delta)=w(X=Y, \mu, \nu, 0),
$$

which is marginal distribution depending on three variables. The physical meaning of the parameters $\mu ; \nu ; \delta$ is that they describe ensemble of shifted, rotated and scaled reference frames in which the position $X$ is measured. Formula (25) can be inverted and the Wigner function of the state can be expressed in terms of the marginal distribution [13]

$$
W(q, p)=(2 \pi)^{2} s^{2} \exp (i s X) w_{F}(X, s q, s p, s)
$$

where $w_{F}(X, a, b, s)$ is the Fourier component of the marginal distribution (25) taken with respect to the parameters $\mu ; \nu ; \delta$, namely,

$$
w_{F}(X, a, b, s)=\frac{1}{(2 \pi)^{3}} \int w(X, \mu, \nu, \delta) \exp [-i(\mu a+\nu b+\delta s)] d \mu d \nu d \delta .
$$

In [14], it was shown that for systems with the Hamiltonians

$$
\hat{H}=\frac{\hat{p}^{2}}{2}+V(\hat{q})
$$

the marginal distribution satisfies the quantum time-evolution equation

$$
\dot{w}-\mu \frac{\partial}{\partial \nu} w-i\left[V\left(\frac{1}{\partial / \partial \delta} \frac{\partial}{\partial \mu}+i \frac{\nu}{2} \frac{\partial}{\partial \delta}\right)-V\left(\frac{1}{\partial / \partial \delta} \frac{\partial}{\partial \mu}-i \frac{\nu}{2} \frac{\partial}{\partial \delta}\right)\right] w=0 .
$$

The dot implies partial time derivative. This equation can be rewritten for the function $P$ (26) in the form

$$
\dot{P}-\mu \frac{\partial}{\partial \nu} P-i\left[V\left(\frac{-1}{\partial / \partial Y} \frac{\partial}{\partial \mu}-i \frac{\nu}{2} \frac{\partial}{\partial Y}\right)-V\left(\frac{-1}{\partial / \partial Y} \frac{\partial}{\partial \mu}+i \frac{\nu}{2} \frac{\partial}{\partial Y}\right)\right] P=0 .
$$

The measurable position $Y$ is cyclic variable for the evolution equation. For the trapped ion, Eq. (30) takes the form

$$
\dot{w}-\mu \frac{\partial}{\partial \nu} w+\omega^{2}(t) \nu \frac{\partial}{\partial \mu} w=0
$$

or in terms of the function $P$,

$$
\dot{P}-\mu \frac{\partial}{\partial \nu} P+\omega^{2}(t) \nu \frac{\partial}{\partial \mu} P=0
$$

For squeezed and correlated states of the trapped ion, the Wigner function has the form (匹) in which quadrature variances and covariance for the state (16) are given by (17) and (18) and quadrature means are equal to zero. For the state (15), the dispersion matrix is the same but two quadrature means have nonzero values

$$
\begin{aligned}
& \langle p\rangle=\frac{1}{\sqrt{2}}\left(\alpha \dot{\varepsilon}^{*}+\alpha^{*} \dot{\varepsilon}\right) \\
& \langle q\rangle=\frac{1}{\sqrt{2}}\left(\alpha \varepsilon^{*}+\alpha^{*} \varepsilon\right) .
\end{aligned}
$$


Calculating integral (25) one can show that for generic Gaussian packets of the trapped ion [also, for particular cases (15) and (16) ] the marginal distribution is

$$
w(X, \mu, \nu, \delta, t)=\frac{1}{\sqrt{2 \pi \sigma_{X}(t)}} \exp \left\{-\frac{(X-\bar{X})^{2}}{2 \sigma_{X}(t)}\right\}
$$

where the dispersion of the symplectic observable $X$ and the mean value of the observable depend on time and parameters as follows

$$
\begin{aligned}
\sigma_{X}(t) & =\mu^{2} \sigma_{q q}+\nu^{2} \sigma_{p p}+2 \mu \nu \sigma_{p g} \\
\bar{X} & =\mu\langle q\rangle+\nu\langle p\rangle+\delta .
\end{aligned}
$$

It is the same form of Gaussian distribution discussed for free motion and harmonic oscillator in [14 but with different quadrature dispersions and means which are given by (17), (18) and (34), (35), correspondingly.

One can check that the normalized marginal distribution (36) with parameters (37) and (38) and quadrature dispersions and means (17), (18) and (34), (35) satisfies the evolution equation (32). This follows from the observation that the marginal distribution depends on the symplectic parameters and time only through the dependence on the mean value $\bar{X}$ and dispersion $\sigma_{X}(t)$. Then calculating the first derivatives of the marginal distribution in all variables and using the Ehrenfest theorem for quadrature means and also the evolution equations for variances and covariance of the parametric oscillator [18], one can show that the evolution equation is satisfied by the function (36).

The evolution of the Wigner function of trapped ion (as the system with quadratic Hamiltonian) for any state is given by the following prescription (see, for example, 21]). Given the Wigner function $W(p, q, t=0)$ at the initial moment of time $t=0$. Then the Wigner function at time $t$ is obtained by the replacement

$$
W(p, q, t)=W[p(t), q(t), t=0]
$$

where time-dependent arguments are the linear integrals of motion of the quadratic system. The linear integrals of motion describe initial values of classical trajectories in the phase space of the system

$$
\begin{aligned}
& p(t)=\frac{\varepsilon+\varepsilon^{*}}{2} p-\frac{\dot{\varepsilon}+\dot{\varepsilon}^{*}}{2} q ; \\
& q(t)=\frac{\varepsilon-\varepsilon^{*}}{2 i} p+\frac{\dot{\varepsilon}-\dot{\varepsilon}^{*}}{2 i} q .
\end{aligned}
$$

This ansatz follows from the statement that the density operator of the Hamiltonian system is the integral of motion and its matrix elements in any basis must depend on appropriate integrals of motion. Consequently, for arbitrary mixed state of the ion in the Paul trap with the initial Gaussian Wigner function (4), time evolution of the Wigner function is given by the same formula (4) in which the variables $p$ and $q$ are replaced by the functions (39), correspondingly. 
The marginal distribution is related to the Wigner quasi-distribution function by (25). So, one can extend the above anzats to the marginal distribution. We formulate this anzats for the Paul trap as follows. Given an initial distribution function

$$
w(X, \mu, \nu, \delta, t=0)=w_{0}(X-\delta, \mu, \nu)
$$

Then at time $t \neq 0$, the marginal distribution which is the solution to Eq. (32) has the form

$$
w(X, \mu, \nu, \delta, t)=w_{0}(X-\delta, \mu(t), \nu(t))
$$

in which

$$
\begin{aligned}
& \nu(t)=\frac{\dot{\varepsilon}-\dot{\varepsilon}^{*}}{2 i} \nu+\frac{\varepsilon-\varepsilon^{*}}{2 i} \mu ; \\
& \mu(t)=\frac{\dot{\varepsilon}+\dot{\varepsilon}^{*}}{2} \nu+\frac{\varepsilon+\varepsilon^{*}}{2} \mu .
\end{aligned}
$$

\section{$5 \quad$ Even and Odd Coherent States}

Now we will discuss marginal probability for nonclassical states of the parametric oscillator, such as even and odd coherent states [3]. To describe such nonclassical states, as even and odd coherent states not only for an ion in the Paul trap but also for other types of trapped ions we consider construction of multimode even and odd coherent states (see, for example, 21, 22, ). Symplectic tomography procedure [13] was extended for multimode systems in [23].

We define multimode even and odd coherent states as

$$
\left|\mathbf{A}_{ \pm}\right\rangle=N^{( \pm)}(|\mathbf{A}\rangle \pm|-\mathbf{A}\rangle)
$$

where the multimode coherent state $|\mathbf{A}\rangle$ is

$$
|\mathbf{A}\rangle=\left|\alpha_{1}, \alpha_{2}, \ldots, \alpha_{n}\right\rangle=D(\mathbf{A})|\mathbf{0}\rangle
$$

and the multimode coherent state is created from the multimode vacuum state $|\mathbf{0}\rangle$ by the multimode displacement operator $D(\mathbf{A})$. The definition of multimode even and odd coherent states is the obvious generalization of the one-mode even and odd coherent states. Normalization constants for multimode even and odd coherent states are

$$
\begin{aligned}
N^{(+)} & =\frac{\exp \left(|\mathbf{A}|^{2} / 2\right)}{2 \sqrt{\cosh |\mathbf{A}|^{2}}} ; \\
N^{(-)} & =\frac{\exp \left(|\mathbf{A}|^{2} / 2\right)}{2 \sqrt{\sinh |\mathbf{A}|^{2}}},
\end{aligned}
$$

where $\mathbf{A}=\left(\alpha_{1}, \alpha_{2}, \ldots, \alpha_{n}\right)$ is a complex vector. 
The Wigner function for multimode coherent states is (see [18] )

$$
W_{\mathbf{A}, \mathbf{B}}=2^{N} \exp \left(-2 \mathbf{Z Z}^{*}+2 \mathbf{A} \mathbf{Z}^{*}+2 \mathbf{B}^{*} \mathbf{Z}-\mathbf{A B}^{*}-\frac{|\mathbf{A}|^{2}}{2}-\frac{|\mathbf{B}|^{2}}{2}\right),
$$

where

$$
\mathbf{Z}=\frac{\mathbf{q}+i \mathbf{p}}{\sqrt{2}}
$$

For even and odd coherent states, the Wigner function is (see [22])

$$
\begin{aligned}
W_{\mathbf{A}_{ \pm}}(\mathbf{q}, \mathbf{p}) & =\left|N^{( \pm)}\right|^{2}\left[W_{(\mathbf{A}, \mathbf{B}=\mathbf{A})}(\mathbf{q}, \mathbf{p}) \pm W_{(\mathbf{A}, \mathbf{B}=-\mathbf{A})}(\mathbf{q}, \mathbf{p})\right. \\
& \left. \pm W_{(-\mathbf{A}, \mathbf{B}=\mathbf{A})}(\mathbf{q}, \mathbf{p})+W_{(-\mathbf{A}, \mathbf{B}=-\mathbf{A})}(\mathbf{q}, \mathbf{p})\right]
\end{aligned}
$$

where explicit forms of $N^{( \pm)}$are given by Eq. (45). For multimode case, we use following notation

$$
\begin{aligned}
& \mathbf{A} \mathbf{Z}^{*}=\alpha_{1} Z_{1}^{*}+\alpha_{2} Z_{2}^{*}+\cdots \alpha_{n} Z_{n}^{*} ; \\
& \mathbf{Z Z}^{*}=Z_{1} Z_{1}^{*}+Z_{2} Z_{2}^{*}+\cdots+Z_{n} Z_{n}^{*} .
\end{aligned}
$$

If at time $t=0$, one has the initial Wigner function of the system in the form

$$
W(\mathbf{p}, \mathbf{q}, t=0)=W_{0}(\mathbf{Q}),
$$

the Wigner function of the system at time $t$ is

$$
W(\mathbf{p}, \mathbf{q}, t)=W_{0}[\Lambda(t) \mathbf{Q}+\Delta(t)] .
$$

where the matrix $\Lambda(t)$ and the vector $\Delta(t)$ determine the linear integrals of motion for quadratic systems to which the parametric oscillator belongs [21].

Thus, we get the expressions for the Wigner functions of even and odd coherent states of the trapped ion, if in formulas (48) we put $n=1$ and replace $\mathbf{p}$ and $\mathbf{q}$ by the linear integrals of motion of the parametric oscillator

$$
\begin{aligned}
& p_{0}=\frac{A-A^{\dagger}}{i \sqrt{2}} ; \\
& q_{0}=\frac{A+A^{\dagger}}{\sqrt{2}},
\end{aligned}
$$

where the invariant $A$ is given by (11). The explicit form of the invariants $p_{0} ; q_{0}$ which should be considered as $c$-numbers is given by (39). Since the marginal distribution is related to density matrix which is the integral of motion, the time dependence of the marginal distribution for the parametric oscillator may be given by analogous prescription which was used for the Wigner function. If one knows the marginal distribution at the initial moment of time as the function of 
the parameters $\mu$ and $\nu$, at time $t$ these parameters in the expression for the initial marginal distribution should be replaced by linear combinations of $\mu$ and $\nu$ (42).

The marginal distribution for initial even and odd coherent states was given in [14]

$$
w^{ \pm}(X, \mu, \nu, \delta)=N^{( \pm) 2} \frac{1}{\sqrt{\pi\left(\mu^{2}+\nu^{2}\right)}}\left\{w_{1}+w_{2} \pm w_{3} \pm w_{4}\right\}
$$

where

$$
\begin{aligned}
w_{1} & =\exp \left[-\frac{\left(Y-s-s^{*}\right)^{2}}{\mu^{2}+\nu^{2}}\right] ; \\
w_{2} & =\exp \left[-\frac{\left(Y+s+s^{*}\right)^{2}}{\mu^{2}+\nu^{2}}\right] ; \\
w_{3}=w_{4}^{*} & =\exp \left[-2|\alpha|^{2}-\frac{\left(Y-s+s^{*}\right)^{2}}{\mu^{2}+\nu^{2}}\right] ; \\
Y & =X-\delta ; \\
s & =\sqrt{2} \alpha(\mu-i \nu) .
\end{aligned}
$$

Thus, replacing the parameters $\nu$ and $\mu$ in (53) by time-dependent functions (42) we get the marginal distribution of the trapped ion at any time moment $t$.

The optical tomography procedure suggested in [10, 12] is the partial case of symplectic tomography, since for the optical tomography one has to take the symplectic parameters in the form

$$
\begin{aligned}
\mu & =\cos \phi \\
\nu & =\sin \phi ; \\
\delta & =0 .
\end{aligned}
$$

Thus, the marginal distribution for rotated quadrature in case of initial even and odd coherent states takes the form

$$
w^{ \pm}(X, \varphi, t=0)=N^{( \pm) 2} \frac{1}{\sqrt{\pi}}\left\{w_{1}(X, \varphi)+w_{2}(X, \varphi) \pm w_{3}(X, \varphi) \pm w_{4}(X, \varphi)\right\}
$$

where

$$
\begin{aligned}
w_{1}(X, \varphi) & =\exp \left[-\left(X-2 \sqrt{2}|\alpha| \cos \left(\varphi_{\alpha}-\varphi\right)\right)^{2}\right] ; \\
w_{2}(X, \varphi) & =\exp \left[-\left(X+2 \sqrt{2}|\alpha| \cos \left(\varphi_{\alpha}-\varphi\right)\right)^{2}\right] ; \\
w_{3}(X, \varphi)=w_{4}^{*}(X, \varphi) & =\exp \left[-2|\alpha|^{2}-\left(X-i 2 \sqrt{2} \sin \left(\varphi_{\alpha}-\varphi\right)\right)^{2}\right] ; \\
\alpha & =|\alpha| \exp i \varphi_{\alpha} .
\end{aligned}
$$

This marginal distribution, used in the optical tomography scheme, at time $t \neq 0$ is given by formula (53) in which the variables $\nu$ and $\mu$ are replaced by the variables

$$
\nu(t, \varphi)=\frac{\varepsilon-\varepsilon^{*}}{2 i} \cos \varphi+\frac{\dot{\varepsilon}-\dot{\varepsilon}^{*}}{2 i} \sin \varphi
$$




$$
\mu(t, \varphi)=\frac{\varepsilon+\varepsilon^{*}}{2} \cos \varphi+\frac{\dot{\varepsilon}+\dot{\varepsilon}^{*}}{2} \sin \varphi .
$$

Using (55) and (56) and measuring the marginal distribution of homodyne output the Wigner function of the trapped ion may be reconstructed by means of the Radon transform [10].

Thus, the rule formulated gives the time dependence of both the marginal distribution of rotated quadrature and the marginal distribution of squeezed and rotated quadrature for even and odd coherent states of trapped ion. Replacing $\mu$ and $\nu$ in (53) by $\mu(t)$ and $\nu(t)$ from (42), we get solution to the dynamical equation (30) for the ion in the Paul trap.

\section{Conclusion}

In the present work, we calculated the marginal distribution of symplectic observable (which is generic linear quadrature) for nonclassical states of trapped ion modelled by the parametric quantum oscillator. Measurements of the marginal distribution give the possibility to measure the quantum states. In the case of particular choice of the parameters $\mu=\cos \varphi ; \nu=\sin \varphi$, the measurement is reduced to finding marginal distribution for homodyne output and reconstructing the Wigner function through the Radon transform of optical tomography scheme [10, 12]. The found distribution of generic linear quadrature satisfies the new classical-like equation of quantum dynamics introduced in the symplectic tomography scheme.

\section{Acknowledgments}

This work has been partially supported by the Russian Basic Research Foundation.

The author would like to acknowledge the International Center for Theoretical Physics in Trieste for kind hospitality.

The author thanks Prof. W. Schleich for discussions and for communication of the results of [15] before publication. The author thanks Prof. V. I. Man'ko and Prof. P. Tombesi for fruitfull discussions.

\section{References}

[1] R. L. de Matos Filho and W. Vogel, Phys. Rev. Lett., 76, 608 (1996).

[2] R. J. Glauber, Phys. Rev. Lett., 10, 84 (1963).

[3] V. V. Dodonov, I. A. Malkin, and V. I. Man'ko, Physica, 72, 597 (1974).

[4] B. Yurke and D. Stoler, Phys. Rev. Lett., 57, 13 (1986).

[5] E. Schrödinger, Naturwissenschaften, 23, 844 (1935). 
[6] R. J. Glauber, in: Recent Developments in Quantum Optics, Proceedings of the International Conference on Quantum Optics (Hyderabad, India, January 1991), ed. R. Inguva (Plenum Press, New York, 1993), p. 1; Quantum Measurements in Quantum Optics, Proceedings of the NATO Advanced Research Workshop (Cortina d'Ampezzo, Italy, January 1991), eds. P. Tombesi and D. F. Walls (Plenum Press, New York, 1991), p. 3; Foundations of Quantum Mechanics, Proceedings of the Conference (Santa Fe, NM, USA, May 1991), eds. T. D. Black et al (World Scientific, Singapore, 1992), p. 23; Laser Manipulation of Atoms and Ions, Proceedings of the International School of Physics "Enrico Fermi" Course 118 (Varenna, Italy, July 1992), eds. E. Arimondo et al (North Holland, Amsterdam, 1992), p. 643.

[7] G. Schrade, V. I. Man'ko, W. Schleich, and R. J. Glauber, Quantum Semiclass. Opt., 7, 307 (1995).

[8] K. Husimi, Prog. Theor. Phys., 9, 238 (1953).

[9] I. A. Malkin and V. I. Man'ko, Phys. Lett. A, 32, 243 (1970);

I. A. Malkin, V. I. Man'ko, and D. A. Trifonov, Phys. Rev. D, 2, 1371 (1970).

[10] K. Vogel and H. Risken, Phys. Rev. A, 40, 2847 (1989).

[11] K. E. Cahill and R. J. Glauber, Phys. Rev., 177, 1882 (1969).

[12] D. T. Smithey, M. Beck, M. G. Raymer, and A. Faridani, Phys. Rev. Lett., 70, 1244 (1993).

[13] S. Mancini, V. I. Man'ko, and P. Tombesi, Quantum Semiclass. Opt., 7, 615 (1995).

[14] S. Mancini, V. I. Man'ko, and P. Tombesi, Phys. Lett. A (accepted for publication); "Symplectic tomography as classical approach to quantum systems," Preprint Quant-Ph/9603002.

[15] G. Schrade, P. J. Bardroff, C. Leichte, and W. Schleich, "Endoscopy in the Paul trap" (in preparation).

[16] V. V. Dodonov, E. V. Kurmyshev, and V. I. Man'ko, Phys. Lett. A, 79, 150 (1980).

[17] V. V. Dodonov, O. V. Man'ko, and V. I. Man'ko, Phys. Rev. A, 49, 2993; 50, 813 (1994).

[18] V. V. Dodonov and V. I. Man'ko, Invariants and Evolution of Nonstationary Quantum Systems, Proceedings of the Lebedev Physical Institute, 183 (Nova Science, Commack, New York, 1989).

[19] E. Schrödinger, Sitzungsber. Preuss. Acad. Wiss., 24, 296 (1930).

[20] V. V. Dodonov, V. I. Man'ko, and D. E. Nikonov, Phys. Rev. A, 51, 3328 (1995).

[21] V. I. Man'ko, "Introduction to quantum optics," invited lecture at the XX Latin American School on Physics, Mexico, July 1995 (to be published by World Scientific, Singapore, 1996).

[22] N. A. Ansari and V. I. Man'ko, Phys. Rev. A, 50, 1942 (1994).

[23] G. M. D'Ariano, S. Mancini, V. I. Man'ko, and P. Tombesi, "Reconstructing the density operator by using generalized field quadratures" (submitted to Quantum Semiclass. Opt.). 